\title{
Overproduction of the secretin OutD suppresses the secretion defect of an Erwinia chrysanthemi outB mutant
}

\author{
Guy Condemine and Vladimir E. Shevchik
}

Author for correspondence: Guy Condemine. Tel: +33472 4380 88. Fax: +33472438714.

e-mail: condemin@insa.insa-lyon.fr

Unité Microbiologie et Génétique Composante INSA, UMR CNRS-INSA-UCB 5577, INSA Bat 406, 20 Av Einstein, 69621

Villeurbanne, France
OutB is a component of the Erwinia chrysanthemi Out secretion machinery. Homologues of OutB have been described in two other bacteria, Klebsiella oxytoca and Aeromonas hydrophila, but their requirement in the secretion process seems to be different. Study of OutB topology with the BlaM topology probe suggests that it is an inner-membrane protein with a large periplasmic domain. However, fractionation experiments indicate that it could be associated with the outer membrane through its C-terminal part. The secretion deficiency of an Erw. chrysanthemi outB mutant can be reversed by the addition of an inducer of the $k d g R$ regulon. It was shown that this effect results from the increased expression of the secretin OutD and that secretion can be restored in an outB mutant by introducing the outD gene on a plasmid. Several experiments suggest an interaction between OutB and OutD. In Erw. chrysanthemi, the presence of OutD stabilizes OutB. OutD expressed in Escherichia coli can be protected from proteolytic degradation by the coexpression of OutB. This effect does not require the $\mathbf{N}$-terminal, transmembrane segment of outB. OutB can be cross-linked with OutD by formaldehyde. These results indicate that OutB could act with OutD in the functioning of the Out secretion machinery.

Keywords: general secretory pathway, protein secretion, protein-protein interaction, secretin

\section{INTRODUCTION}

Pectinases, the main virulence factors of the phytopathogenic bacteria Erwinia chrysanthemi, are secreted by a type II secretion system. Such type II machineries [or the main terminal branch of the general secretory pathway (GSP)] are found in several other Gramnegative bacteria that secrete toxins or lytic enzymes in the outer medium: Erwinia carotovora, Pseudomonas aeruginosa, Aeromonas hydrophila, Klebsiella oxytoca and Vibrio cholerae (Pugsley, 1993). Secreted enzymes are synthesized with an N-terminal signal sequence, which is cleaved during the Sec-dependent translocation across the inner membrane. In the absence of the GSP machinery, these proteins remain in the periplasm. Biogenesis and functioning of the various GSP machineries requires the products of a minimum of 12

Abbreviations: CMC, carboxymethylcellulose; GSP, general secretory pathway; PGA, polygalacturonate genes ( $g s p C-M$ and $g s p O)$ in $P$. aeruginosa but this number can go up to 15 in Erw. chrysanthemi or Erw. carotovora. Little is known about the function of their products in protein secretion. GspD is a member of a family of outer-membrane proteins, named secretins, involved in the traffic of macromolecules across the outer membrane. The filamentous phage $\mathrm{f} 1 \mathrm{pIV}$ protein, Yersinia enterocolitica YscC and P. aeruginosa XcpQ and PilQ are secretins that form homomultimeric rings which could constitute the pore through which phages or proteins would cross the outer membrane (Linderoth et al., 1997; Bitter et al., 1998). The N-terminal part of the secretins could also play a role in the selection of proteins to secrete. Erw. chrysanthemi OutD interacts, through its $\mathrm{N}$-terminal domain, with secreted proteins and this interaction could be the key to the specificity of the secretion mechanism (Shevchik et al., 1997). The Ntermini of GspG, H, I, J and K resemble that of the type IV pilin subunit and thus these proteins are designated pseudopilins (Bleves et al., 1998). Their leader peptide is cleaved by the GspO prepilin peptidase (Nunn \& Lory, 
1991). GspE is an ATP-binding protein with a kinase activity (Sandkvist et al., 1995). The bitopic innermembrane proteins GspC, $\mathrm{L}$ and $\mathrm{M}$ have a large periplasmic domain and GspF is a polytopic innermembrane protein (Reeves et al., 1994; Thomas et al., 1997). Some possible interactions between components of the GSP machinery have been described: OutE-OutL, EspE-EspL, XcpT-XcpR, XcpY-XcpZ and EpsLEpsM (Py et al., 1999; Sandkvist et al., 1995, 1999; Kagami et al., 1998; Michel et al., 1998).

Besides this conserved core of 12 proteins, some GSP proteins are found in only a few bacteria. GspN has been found in Erw. carotovora but not in Erw. chrysanthemi (Lindeberg \& Collmer, 1992). GspA, a protein with putative ATPase activity, has only been detected in Escherichia coli and A. hydrophila (exeA) (Francetic \& Pugsley, 1996; Jahagirdar \& Howard, 1994). GspS has only been identified in K. oxytoca, in the enterohaemorrhagic Esc. coli O157:H7 strain, in Erw. chrysanthemi and Erw. carotovora (PulS, EtpO and OutS) (d'Enfert \& Pugsley, 1989; Schmidt et al., 1997; Condemine et al., 1992; Lindeberg et al., 1996). These GspS proteins are outer-membrane lipoproteins that stabilize the secretins PulD and OutD and help their insertion in the outer membrane (Hardie et al., 1996; Shevchik \& Condemine, 1998). The interaction requires a 62 aa domain present at the C-terminal extremity of PulD and OutD (Daefler et al., 1997; Shevchik \& Condemine, 1998). This domain is also present in the Erw. carotovora OutD protein but not in other secretins. Thus, GspS probably does not exist in other bacteria. GspB has been described in three bacteria: K. oxytoca, Erw. chrysanthemi and A. hydrophila (d'Enfert \& Pugsley, 1989; Condemine et al., 1992; Jahagirdar \& Howard, 1994). It probably also exists in Erw. carotovora (Lindeberg et al., 1996). In the first two bacteria, the gene $g s p B$ is clustered with $g s p S$, whereas in $A$. hydrophila exeB forms an operon with exeA. ExeB forms a complex with the ATPase ExeA (Schoenhofen et al., 1998) and is required for efficient secretion of aerolysin in this bacterium. By contrast, a K. oxytoca pulB mutant has no phenotype. In Erw. chrysanthemi, the absence of OutB leads to an intermediate phenotype, with secretion of pectate lyase reduced by $30 \%$. In this study, we analysed the cellular localization of OutB and showed that a mutation in outB can be suppressed by overproduction of OutD. Our results suggest that OutB interacts with OutD.

\section{METHODS}

Bacterial strains, plasmids, culture conditions and plate assays. Bacterial strains are described in Table 1. Erw. chrysanthemi and Esc. coli cells were grown at $30^{\circ} \mathrm{C}$ and $37^{\circ} \mathrm{C}$, respectively, in Luria-Bertani (LB) medium or M63 minimal medium (Miller, 1972), supplemented with a carbon source $[0.2 \%$, except polygalacturonate (PGA) $0.08 \%]$ and, when required, with amino acids $\left(40 \mu \mathrm{g} \mathrm{ml}^{-1}\right)$ and antibiotics at the following concentrations: ampicillin, $100 \mu \mathrm{g} \mathrm{ml}^{-1}$; kanamycin, $50 \mu \mathrm{g} \mathrm{ml}^{-1}$; chloramphenicol, $20 \mu \mathrm{g} \mathrm{ml}^{-1}$. LB medium was solidified with $12 \mathrm{~g}$ agar ${ }^{-1}$ to give GL plates. Pectate lyase activity was revealed by flooding PGA-containing agar plates with a saturated solution of copper acetate (Andro et al., 1984). Degraded PGA formed a clear halo around the colonies. Cellulase activity was detected on carboxymethylcellulose (CMC)-containing plates by flooding with $0 \cdot 1 \%$ Congo red for 10 min followed by bleaching with $1 \mathrm{M} \mathrm{NaCl}$ (Wood, 1980).

Recombinant DNA techniques. Preparation of plasmid DNA, restriction, ligation, DNA electrophoresis, transformation and electroporation were carried out as described by Sambrook $e t$ al. (1989). Nucleotide sequence analysis was performed using the Pharmacia T7 sequencing kit. To construct the periplasmic derivative of OutB (PelB $\left.{ }^{\mathrm{sp}}-\mathrm{OutB}\right)$, the EcoR V-EcoRI fragment from plasmid pBT1 carrying outB and outS was cloned between the EcoRV and EcoRI sites of pET-20b $(+)$ under the PelB signal peptide. The pelB $B^{\text {sp }}$ out $B$ construct was then digested with BglII and XhoI and introduced into the expression vector pACT3 digested by BamHI and SalI. Finally, the out $S$ gene was deleted from the construct by digestion with HindIII and EcoNI, treatment with the Klenow enzyme and ligation to give plasmid pABSC3.

Construction of OutB-BlaM protein fusions. The outB gene was cloned between the EcoRI and KpnI sites of plasmid pJBSKpn, a derivative of plasmid pJBS633 (Broome-Smith et al., 1990) that contains an additional KpnI site upstream of the blaM gene. The resulting construct was digested with $K p n \mathrm{I}$ and HindIII that cleaves the plasmid downstream of outB to produce exonuclease III-insensitive and sensitive sites, respectively. Deletions in outB were generated with the Exonuclease III deletion kit (USB). After ligation and transformation, the $\mathrm{Kan}^{\mathrm{r}}$ clones obtained were first replicated on GL plates containing Amp $\left(50 \mu \mathrm{g} \mathrm{ml}^{-1}\right)$ to identify those producing in-frame OutB-BlaM fusion proteins. Four Amp ${ }^{r}$ clones were retained. For these, the MIC of ampicillin required to prevent colony formation by a single cell was determined and nucleotide sequencing was performed to characterize the outB-blaM junction.

OutB purification. The periplasmic soluble form of OutB (PelB $\left.{ }^{\mathrm{sp}}-\mathrm{OutB}\right)$ was overproduced in the NM522/pABSC3 strain. Cells were grown in $100 \mathrm{ml} \mathrm{LB}$ containing chloramphenicol. At an $\mathrm{OD}_{600}$ of 0.5 , IPTG was added to $1 \mathrm{mM}$ and, after $2 \mathrm{~h}$ additional growth, the cells were harvested by centrifugation for $5 \mathrm{~min}$ at $5000 \mathrm{~g}$ and frozen at $-80^{\circ} \mathrm{C}$. The overproduced protein was extracted from cells by three cycles of freeze-thawing (Johnson \& Hecht, 1994). The supernatant was loaded onto a $15 \%(\mathrm{w} / \mathrm{v})$ preparative SDS-PAGE. The band containing PelB $\mathrm{B}^{\mathrm{sp}}-\mathrm{OutB}$ was cut out and the protein was extracted by three washes with $10 \mathrm{mM}$ Tris/ $\mathrm{HCl} \mathrm{pH} 8 \cdot 0$, $0 \cdot 1 \%$ SDS. The protein was concentrated and injected into a rabbit for antibody production by Valbex (Villeurbanne).

Protein labelling. Overexpression and exclusive labelling of plasmid-encoded proteins was carried out using the T7 promoter/T7 polymerase system of Tabor \& Richardson (1985).

Gel electrophoresis and immunoblotting. SDS-PAGE was usually performed according to Laemmli (1970). Proteins were transferred onto nitrocellulose in a semi-dry apparatus and the membrane was incubated with antibodies and developed with the ECL detection kit (Amersham), as described previously (Shevchik et al., 1996). The primary antibodies used were antiOutB diluted 1:5000, anti-BlaM diluted 1:5000, anti-OutD diluted 1:3000 and anti-EGZ diluted 1:3000.

Isolation and analysis of cell fractions. Exponentially growing 
Table 1. Bacterial strains and plasmids used in this study

\begin{tabular}{|c|c|c|}
\hline Strain/plasmid & Genotype/description & Reference/origin \\
\hline \multicolumn{3}{|l|}{ Strain } \\
\hline \multirow{2}{*}{\multicolumn{3}{|c|}{$\begin{array}{l}\text { Erw. } \\
\text { chrysanthemi }\end{array}$}} \\
\hline & & \\
\hline A350 & $\operatorname{lm} r T^{\mathrm{c}} \operatorname{lac} Z$ & $\begin{array}{l}\text { Hugouvieux-Cotte-Pattat \& } \\
\text { Robert-Baudouy (1985) }\end{array}$ \\
\hline A837 & $\operatorname{lm} r T^{\mathrm{e}} \operatorname{lac} Z k d g R$ & $\begin{array}{l}\text { Condemine \& Robert-Baudouy } \\
\text { (1987) }\end{array}$ \\
\hline A1524 & $\operatorname{lm} r T^{\mathrm{e}}$ lacZ pecS: : MudIPR13 & Reverchon et al. (1994) \\
\hline A1903 & $\operatorname{lm} r T^{\mathrm{c}}$ lacZ outS: :uidA-kan & Condemine et al. (1992) \\
\hline A1919 & $\operatorname{lm} r T^{\mathrm{c}}$ lacZ outC: : uidA-kan & Laboratory collection \\
\hline A2348 & $\operatorname{lm} r T^{\mathrm{c}} \operatorname{lac} Z$ kdgR pecS:: Cm & Laboratory collection \\
\hline A2582 & $\operatorname{lm} r T^{\mathrm{c}}$ lac $Z$ outB: : uidA-kan & Laboratory collection \\
\hline A2591 & $\operatorname{lm} r T^{\mathrm{c}} \operatorname{lac} Z$ outD & Shevchik et al. (1997) \\
\hline A3157 & $\operatorname{lm} r T^{\mathrm{e}} \operatorname{lac} Z$ kdgR outB::uidA-kan & This work \\
\hline A3158 & $\begin{array}{l}\operatorname{lm} r T^{\mathrm{c}} \text { lacZ pecS: : MudIPR13 outB: : uidA- } \\
\text { kan }\end{array}$ & This work \\
\hline A3494 & As A2348 but also outD & This work \\
\hline A3558 & $\operatorname{lm} r T^{\mathrm{c}}$ lacZ outD & This work \\
\hline \multicolumn{3}{|l|}{ Esc. coli } \\
\hline NM522 & $\begin{array}{l}\text { supE thi-1 } \Delta(\text { lac-proAB }) \Delta(\text { mcrB-hsdSM }) 5 \\
\left(\mathrm{r}_{\mathrm{k}}^{-} \mathrm{m}_{\mathrm{k}}^{-}\right)\left(\mathrm{F}^{\prime} \text { proAB lacl }{ }^{\mathrm{a}} \Delta \text { lacZM15) }\right.\end{array}$ & Stratagene \\
\hline K38 & $\begin{array}{l}\mathrm{HfrC}(\lambda) \text { phoA4 pit-10 fhuA22 ompF } 627 \\
\text { relA1 }\end{array}$ & Russel \& Model (1984) \\
\hline \multicolumn{3}{|l|}{ Plasmids } \\
\hline pT7-5 & $\mathrm{Ap}^{\mathrm{r}}, \phi 10$ promoter & Tabor \& Richardson (1985) \\
\hline pACT3 & $\mathrm{Cm}^{\mathrm{r}}, \mathrm{ptac}$ & Dykxhoorn et al. (1996) \\
\hline pABTC2 & pACT3 carrying the outB gene & This work \\
\hline pABSC3 & pACT3 carrying the pelB ${ }^{\mathrm{sp}}-$ outB construct & This work \\
\hline pBSAC & $\begin{array}{l}\text { pACYC184 carrying outB and outS (1853 } \\
\text { bp) }\end{array}$ & This work \\
\hline pBT1 & pT7-5 carrying outB and outS & This work \\
\hline pET-20b $(+)$ & $\mathrm{Ap}^{\mathrm{r}}$ & Novagen \\
\hline pODN1 & $\begin{array}{l}\text { pBS carrying outD with a AviII-MscI } \\
\text { deletion }\end{array}$ & Shevchik et al. (1997) \\
\hline pTdB-OD & $\begin{array}{l}\mathrm{pT} 7-6 \text {, carrying outD under the pelC } \\
\text { promoter }\end{array}$ & Shevchik et al. (1997) \\
\hline pJBSkpn & $\operatorname{kan}^{\mathrm{r}}$, blaM & $\begin{array}{l}\text { J. C. Lazzaroni, Claude Bernard } \\
\text { University, Villeurbanne, France }\end{array}$ \\
\hline
\end{tabular}

cells $\left(\mathrm{OD}_{600} 0 \cdot 8-1 \cdot 0\right)$ were used for cell fractionation. Cellmembrane fractionation was performed by sucrose gradient centrifugation, as previously described (Shevchik et al., 1996). NADH oxidase was assayed as described by Osborn et al. (1972). Crude membrane fractions were isolated by centrifugation ( $200000 \mathrm{~g}$ for $2 \mathrm{~h}$ ) after French press disintegration of cells and resolubilized in $50 \mathrm{mM}$ Tris $/ \mathrm{HCl} \mathrm{pH} \mathrm{8 \cdot 0.}$

Cross-linking experiments. Bacteria were grown in LB medium to an $\mathrm{OD}_{600}$ of $1 \cdot 0$. An aliquot of $1 \mathrm{ml}$ of culture was centrifuged, the bacteria were washed in $10 \mathrm{mM}$ potassium phosphate buffer $\mathrm{pH} 6.8$ and resuspended in the same volume of the buffer. The cells were incubated with $1 \%$ formaldehyde at room temperature for $30 \mathrm{~min}$, washed in phosphate buffer, and resuspended in SDS sample buffer for analysis by SDSPAGE and immunoblotting.

\section{RESULTS}

\section{OutB encodes a protein with an apparent molecular mass of $29 \mathrm{kDa}$}

During the course of our work, we noticed that a C residue had been omitted at position 1235 of the published sequence of outB (GenBank accession number X65265; Condemine et al., 1992). The resulting frameshift has led to the assignment of an erroneous start codon. Considering the corrected sequence, the outB ORF begins at a GTG codon at nucleotide 1370 which is preceded by a putative Shine-Dalgarno sequence (GAGG) at nucleotides 1377-1380. This ORF encodes a putative protein of 220 amino acids with a deduced molecular mass of $23489 \mathrm{Da}$. To detect the protein 


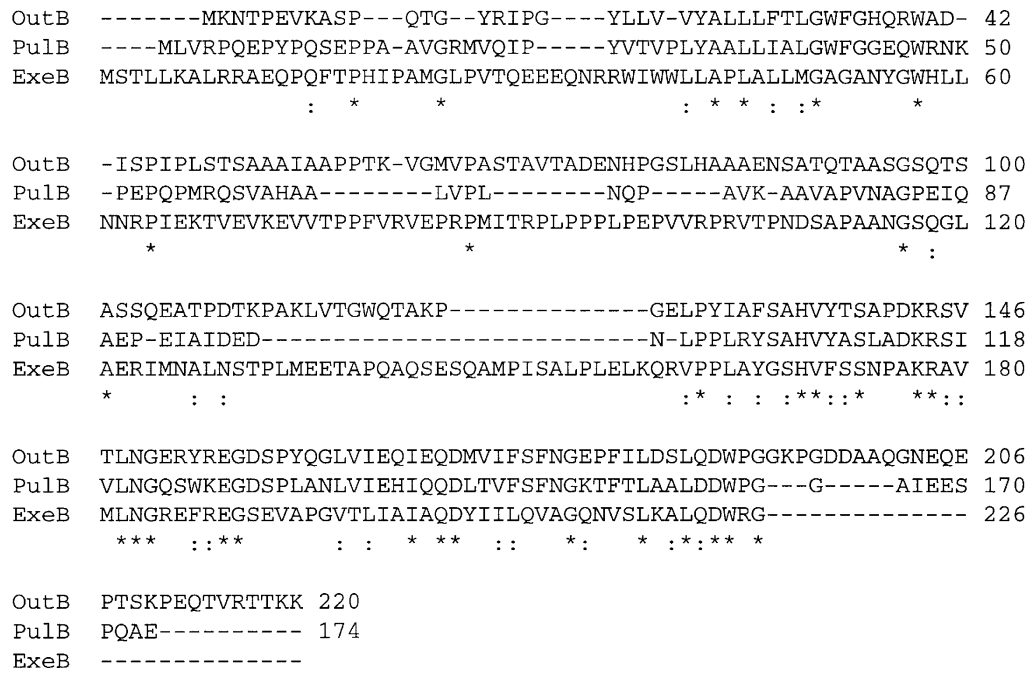

\begin{abstract}
Fig. 1. Alignment of the sequences of OutB, PulB and ExeB with the CLUSTAL program. The stars indicate conserved residues, double dots indicate chemically equivalent residues. The sequence of PulB is from d'Enfert \& Pugsley (1989) and the sequence of ExeB from Jahagirdar \& Howard (1994).
\end{abstract}

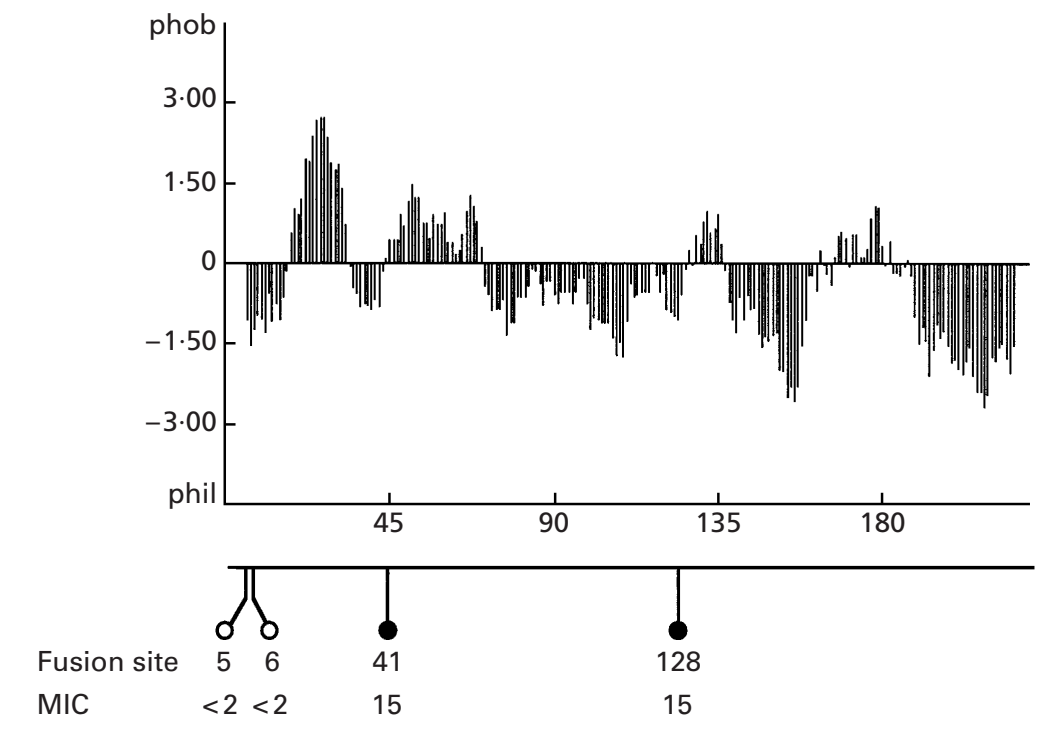

Fig. 2. Topological analysis of OutB. Hydrophobicity profile of OutB using the method of Kyte \& Doolittle (1982). The position of $\mathrm{Amp}^{\mathrm{s}}$ (open circles) and $\mathrm{Amp}^{\mathrm{r}}$ (filled circles) BlaM fusion points are shown beneath the hydrophobicity profile with the number of the corresponding residue. The MIC for each fusion is indicated.

encoded by this ORF, the $1.8 \mathrm{~kb} B c l \mathrm{I}-\mathrm{N}$ siI fragment containing this ORF was cloned under the T7 promoter of plasmid pT7-5. Exclusive labelling of the proteins encoded by this DNA fragment with $\left[{ }^{35} \mathrm{~S}\right]$ methioninecysteine revealed a protein with an apparent molecular mass of $29 \mathrm{kDa}$ (data not shown). The anomalous migration of OutB could result from its amino acid composition since it contains $9 \%$ of proline, which could modify its mobility in SDS-polyacrylamide gels. Such an aberrant mobility has been described for ExeB (12\% proline) and the proline-rich protein TonB $(16 \%$ proline) (Howard et al., 1996).

\section{Topological analysis of OutB}

Alignment of the sequence of OutB with those of PulB and ExeB shows that the three proteins present a low homology throughout their length with some more highly conserved regions in their C-terminal part (Fig. 1). Their hydropathy profiles, analysed by the method of
Kyte \& Doolittle (1982), are also very similar. In their $\mathrm{N}$-terminal parts, they contain, after a short hydrophilic segment, a highly hydrophobic region of about 20 residues which does not present the features of a signal sequence but which could anchor the protein in the cytoplasmic membrane (Fig. 2). To elucidate the OutB topology, we constructed in-frame fusions between OutB and the topology probe $\beta$-lactamase. Four inframe OutB-BlaM fusions were obtained with the fusion at residues 5, 6, 41 and 128 of OutB. The first two gave a MIC $<2 \mu \mathrm{g} \mathrm{ml}^{-1}$ to isolated Esc. coli colonies on GL Amp plates whereas the last two gave an MIC of $15 \mu \mathrm{g} \mathrm{ml}^{-1}$. This suggests that OutB possesses a short Nterminal cytoplasmic extremity, a transmembrane segment and a large C-terminal periplasmic domain. The same MICs were obtained when the constructs were introduced into Erw. chrysanthemi strain A350, in an outS mutant (A1903), or in a polar outC mutant (A1919) that lacks all the other Out proteins, confirming that the topology of the OutB-BlaM fusions is the same in Erw. 
Sucrose (\%) 65

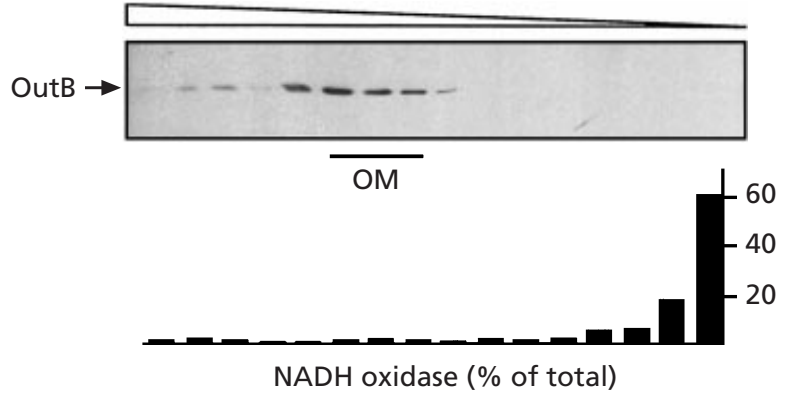

Fig. 3. Cellular localization of OutB. The membrane fraction of Erw. chrysanthemi strain A350 was separated in a flotation sucrose gradient. An aliquot from each fraction was analysed by SDS-PAGE and immunoblotting with anti-OutB or assayed for NADH oxidase activity. The position of the porins, visualized by Ponceau red staining of the membrane, was used to determine the position of outer-membrane-containing fractions.

chrysanthemi as in Esc. coli and shows that no other Out protein is able, by interacting with the fusions, to modify the MIC produced.

Other experiments were performed to confirm the localization of OutB. When the membranes of the Erw. chrysanthemi strain A350 were separated by sucrose density gradient centrifugation, either by flotation or by sedimentation, OutB was not found in the NADHoxidase-containing fractions (inner membrane) but in fractions corresponding to the outer membrane (Fig. 3). The same result was observed in an outS mutant, in a mutant containing a polar mutation in out $C$, or when OutB was produced in Esc. coli (data not shown), indicating that no other Out protein is required for the proper localization of OutB. A large degradation product of OutB $(20 \mathrm{kDa})$ was found in the periplasmic fraction during cell fractionation experiments (data not shown). Taken together, the results obtained with the BlaM topology probe and with membrane fractionation suggest that OutB is an inner-membrane protein with a large periplasmic domain that could interact with the outer membrane. The interaction with the outer membrane seems to be stronger since it resists cell breakage and membrane separation.

\section{Overproduction of OutD phenotypically suppresses an outB mutation}

The effect of an out $B$ mutation on pectate lyase secretion varies depending on the growth conditions used, mainly the presence or absence of pectate lyase synthesis inducers. To clarify this effect, we studied the secretion of the cellulase EGZ, which is constitutively expressed, and of pectate lyases, whose synthesis strongly increases in the presence of galacturonate or PGA. These compounds also induce the synthesis of OutC-O proteins (Condemine \& Robert-Baudouy, 1995). However, PGA has to be degraded by pectinases produced at a basal
Table 2. Secretion of pectate lyases and EGZ in outB mutants

Secretion was estimated by the size of substrate degradation haloes on $0.08 \%$ PGA + glycerol or LB + CMC agar plates after 16 h growth. Halo size: $+/-,<1 \mathrm{~mm} ;+, 1-3$ $\mathrm{mm} ;++, 3-5 \mathrm{~mm} ;+++,>5 \mathrm{~mm}$. Galacturonate $(0 \cdot 2 \%)$ was added as inducer.

\begin{tabular}{|c|c|c|c|c|c|}
\hline \multirow[t]{2}{*}{ Strain } & \multirow{2}{*}{$\begin{array}{l}\text { Genotype } \\
\text { Inducer... }\end{array}$} & \multicolumn{2}{|c|}{ Pectate lyase } & \multicolumn{2}{|c|}{ EGZ } \\
\hline & & - & + & - & + \\
\hline $\begin{array}{l}\text { A350 } \\
\text { A2582 } \\
\text { A3157 } \\
\text { A3158 } \\
\text { A2582/ } \\
\text { pTdB-OD }\end{array}$ & $\begin{array}{l}\text { WT } \\
\text { outB } \\
\text { outB } k d g R \\
\text { outB pecS }\end{array}$ & $\begin{array}{c}+++ \\
+ \\
+++ \\
+++ \\
+++\end{array}$ & $\begin{array}{l}+++ \\
+++ \\
+++ \\
+++ \\
+++\end{array}$ & $\begin{array}{l}+++ \\
+/- \\
+++ \\
+++ \\
+++\end{array}$ & $\begin{array}{l}+++ \\
+++ \\
+++ \\
+++ \\
+++\end{array}$ \\
\hline
\end{tabular}

level by the bacteria to generate inducing molecules. Thus, induction by PGA is strongly concentration dependent. Using a low concentration of PGA in the plates $\left(0 \cdot 8 \mathrm{~g} \mathrm{l}^{-1}\right)$, we could detect pectate lyase activity without inducing too strongly their synthesis. On glycerol + PGA plates, a small halo generated by secreted pectate lyase was seen around the outB mutant A2582 (Table 2). On glycerol + galacturonate + PGA plates, an outB mutant had a wild-type phenotype for pectate lyase secretion (Table 2). To eliminate the problems of the pectate lyase induction level, we also tested secretion of the cellulase EGZ, whose synthesis is independent of the presence of PGA and galacturonate. EGZ secretion by the outB mutant was almost undetectable on LB + CMC plates. Addition of galacturonate to this medium restored EGZ secretion to a wild-type level (Table 2). This led us to suppose that addition of galacturonate induced the synthesis of a protein which could suppress the outB mutation phenotype. $\mathrm{KdgR}$ is the repressor of the pectinolytic enzyme genes which responds to 2-keto-3-deoxygluconate, the inducer formed from galacturonate. In a $k d g R$ outB mutant (A3158), EGZ secretion was at the wild-type level on LB + CMC medium (Table 2). PecS is another regulator controlling pectinolytic enzyme gene expression. In a pecS outB mutant, EGZ secretion was also restored. Thus, the increased expression of a gene regulated by $k d g R$ and $p e c S$ can suppress the effect of an out $B$ mutation. These two regulators co-repress the expression of the out $\mathrm{C}-\mathrm{O}$ operon [each by a factor of three (Condemine \& Robert-Baudouy, 1995)] and of pectate lyase genes. Since it seemed unlikely that overexpression of pectate lyases could restore EGZ secretion, we introduced plasmids containing various out genes into an outB mutant. Plasmids bearing outD (e.g. pTdB-OD, Table 2) were able to restore EGZ secretion on LB + CMC plates. Analysis of EGZ secretion in liquid medium cultures confirmed these results. Whilst it is fully secreted in the wild-type strain A350, half of EGZ was retained in the cell fraction in the 


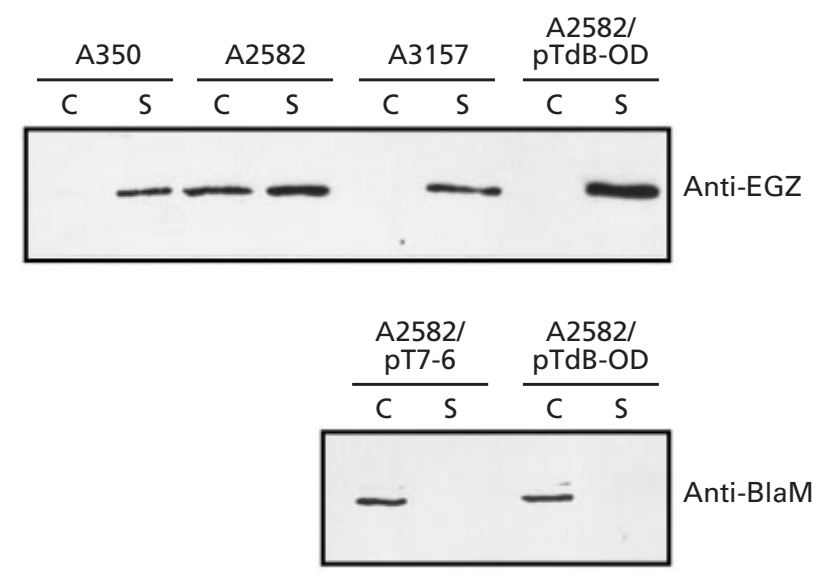

Fig. 4. Secretion of $E G Z$ in outB mutants. Bacteria were grown to an $\mathrm{OD}_{600}$ of 1 in LB medium, centrifuged and resuspended in the same volume of medium. The supernatant $(S)$ and the cell fraction (C) were loaded into a SDS-PAGE and immunoblotted with anti-EGZ (top panel) or anti-BlaM (bottom panel).

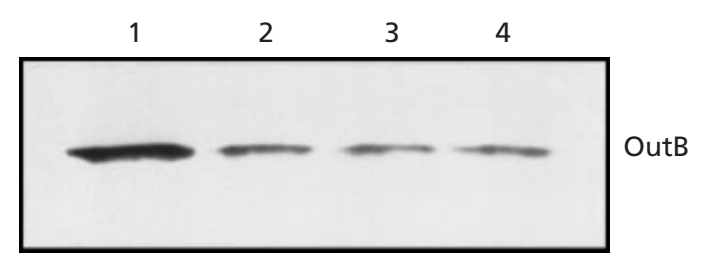

Fig. 5. Amount of OutB in various Erw. chrysanthemi out mutants. Cells were grown overnight in LB medium, proteins were separated by SDS-PAGE and analysed by Western blotting using OutB antibodies. Lanes: 1, A350 (wild-type); 2, A1919 (outC); 3, A3558 (DoutD); 4, A1903 (outS).

outB mutant A2582 (Fig. 4). Complete secretion of EGZ by this strain could be restored by the introduction of a $k d g R$ mutation or of a plasmid bearing outD (Fig. 4). Secretion of EGZ in a strain overexpressing OutD did not result from non-specific leakage, since no $\beta$ lactamase was found in the culture supernant (Fig. 4). The fact that PGA induces OutD synthesis explains why an Out $^{-}$phenotype can not be seen for an outB mutant on medium containing the usual concentration of PGA $\left(4 \mathrm{~g} \mathrm{l}^{-1}\right)$.

\section{Mutual stabilization of OutD and OutB}

Interactions between proteins often lead to their mutual stabilization. To investigate whether OutB interacts with any other Out protein, the amount of OutB in the wild-type strain A350 and in various out mutants was estimated by Western blotting. The quantity of OutB was reduced in the polar outC mutant A1919 (Fig. 5), indicating that some components of the Out machinery stabilize OutB. Since a similar reduction was observed in the non-polar outD mutant A3558, this component might be OutD. To confirm this hypothesis, we checked

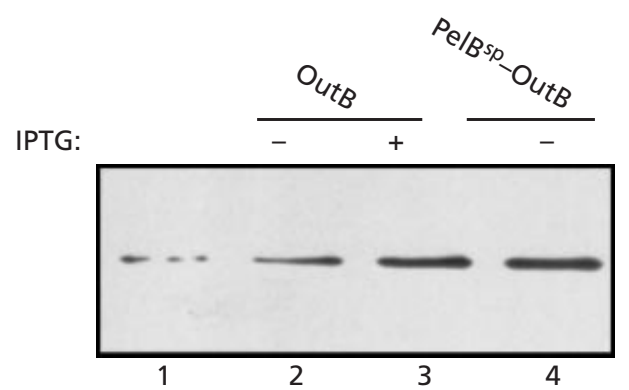

Fig. 6. Stabilization of OutD by OutB. Immunological detection of OutD in Esc. coli NM522 strain carrying pTdB-OD (outD) and either pACT3 (lane 1), pABTC2 (outB) (lanes 2 and 3), or pABSC3 (pe/B $B^{\text {sp }}$-outB) (lane 4). Where indicated, IPTG was added.

the presence of OutB in an outS mutant. In the absence of its chaperone OutS, the amount of OutD is very reduced in Erw. chrysanthemi. The amount of OutB in the outS mutant A1903 was reduced and equivalent to that in the outD mutant (Fig. 5). Remarkably, the amount of OutB in a polar outC mutant was not restored by the introduction of a plasmid bearing outD (results not shown). Thus, the presence of a functional Out machinery might be necessary for OutD to be in a conformation that allows its interaction with OutB. No decrease in the amount of OutD was observed in an outB Erw. chrysanthemi strain, probably because OutD is very stable once inserted in the outer membrane. However, our previous experiments have shown that OutD is very sensitive to proteolytic degradation when expressed in Esc. coli and that it can be stabilized by interaction with the outer-membrane chaperone OutS or with one of the secreted proteins (Shevchik et al., 1997). To test if OutB could protect OutD, we used an Esc. coli strain containing plasmid pABTC2 in which $o u t B$ is expressed from the inducible tac promoter. The amount of OutD increased in the presence of OutB (Fig. 6). This effect was increased when the quantity of OutB was raised by addition of IPTG to the culture. To test if the periplasmic domain of OutB was sufficient to stabilize OutD, we constructed a derivative in which the hydrophobic transmembrane domain of OutB was replaced by the PelB signal sequence. A stabilization of OutD was also observed when the periplasmic form of OutB, PelB ${ }^{\mathrm{sp}}-\mathrm{OutB}$, was produced in trans, indicating that the hydrophobic N-terminal segment of OutB is not required for this effect (Fig. 6, lane 4). Attempts at ligand blotting experiments (binding of labelled OutB to OutD bound onto a nitrocellulose membrane) were unsuccessful, probably because the mutual affinity of the two proteins is not high enough.

\section{Cross-linking of OutB and OutD}

The interaction between OutB and OutD was confirmed by cross-linking experiments. When an Esc. coli strain containing a plasmid bearing outB was treated with formaldehyde, only one protein of the size of OutB was 

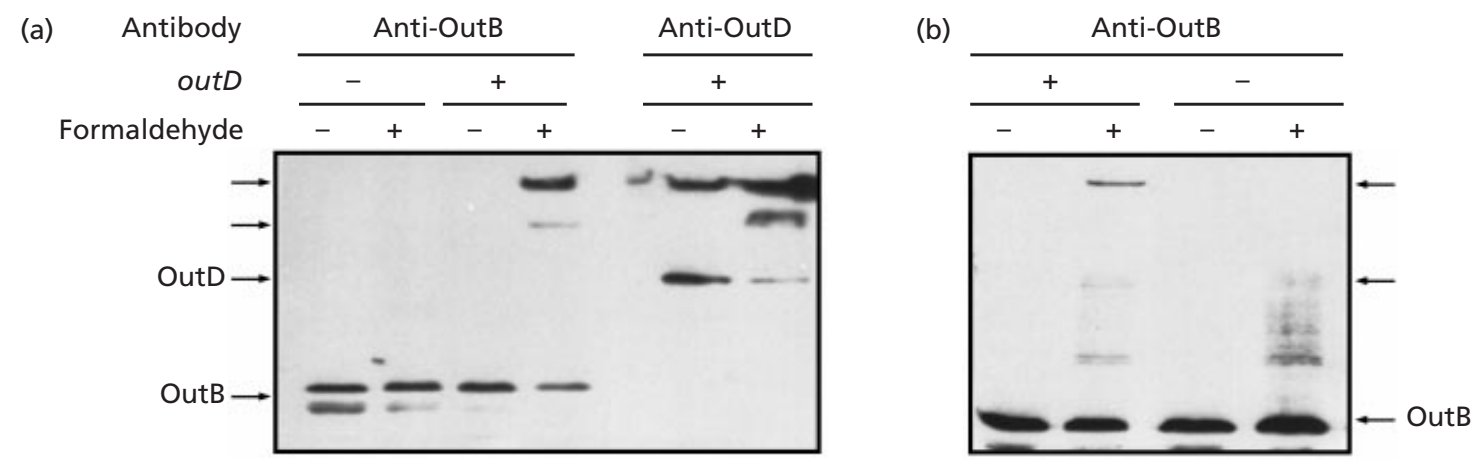

Fig. 7. Detection of formaldehyde-cross-linked OutB by SDS-PAGE and immunoblotting with anti-OutB or anti-OutD. (a) The strains used were NM522(pBSAC/pT7-6) (OutB ${ }^{+}$, OutD ${ }^{-}$) and NM522(pBSAC/pTdB-OD) (OutB ${ }^{+}$, OutD ${ }^{+}$). (b) The strains used are Erw. chrysanthemi A2349 ( $k d g R$ pecS) and A3494 ( $k d g R$ pecS outD). The arrows indicate the position of OutB, OutD and of the cross-linked products.

detected with OutB antibodies (Fig. 7a). When the same experiment was performed with an Esc. coli strain containing the genes outB and outD, additional products reacting with both OutB and OutD antibodies, which could correspond to OutB-OutD cross-linking products, were detected (Fig. 7a). One of these products was detected in the wells of the gel. OutD, like other secretins, forms multimers that are not dissociated in SDS sample buffer unless the samples are boiled for a long time and that stay in the wells of the gel (Shevchik et al., 1997). The same profile was obtained when crosslinking experiments were performed with an Erw. chrysanthemi kdgR pecS mutant A2348 (to maximize OutD synthesis) but not with the outD derivative A3494 (Fig. 7b). Thus, formaldehyde seems able to cross-link OutB with OutD.

\section{DISCUSSION}

In this study, we have analysed the membrane localization and topology of OutB and shown its necessity for type II secretion in Erw. chrysanthemi. Comparison of the hydropathy profile of OutB with those of its two homologues PulB and ExeB, showing an $\mathrm{N}$-terminal hydrophobic segment, led us to suppose that they may have a bitopic conformation with a large periplasmic domain. Some of the experiments presented here confirm this hypothesis. Study of OutB topology with the BlaM topology probe indicated that the $\mathrm{N}$ terminal part of OutB is anchored in the inner membrane and has a small $\mathrm{N}$-terminal cytoplasmic region whilst the region extending after the hydrophobic domain is periplasmic. The low level of resistance observed for the cytoplasmic and the periplamic fusions $\left(2 \mu \mathrm{g} \mathrm{ml}^{-1}\right.$ and $15 \mu \mathrm{g} \mathrm{ml}^{-1}$, respectively) does not result from instability of the fusions but probably from the low level of expression of the outB gene from the plasmid used. The topology of OutB is the same as that proposed for ExeB on the basis of PhoA fusion topology analysis (Howard et al., 1996).

However, OutB presents some unusual properties. When the membranes were separated by sucrose gradi- ent fractionation, OutB was not found in the cytoplasmic-membrane fractions but in higher density fractions that contain outer-membrane proteins. This interaction is strong enough to survive the fractionation procedures. These results may indicate that OutB is, in vivo, anchored in the inner membrane but that its large periplasmic domain allows for an interaction of its Cterminal part with the outer membrane. This interaction would not require an additional Out protein since the separation of OutB with the outer-membrane fractions is not modified by the presence or absence of other Out proteins. Such an association with the outer membrane of a protein predicted to be anchored in the inner membrane has been described for the pseudopilin PulG (Pugsley \& Possot, 1993), PulC (Possot et al., 1999) and for ExeB (Howard et al., 1996). The repeated observation of the presence of ExeB in the outer-membrane fractions has been considered as a contamination (Howard et al., 1996) but this could be a characteristic of the members of the GspB family. The presence of some GSP components, such as PulC and PulG, in both membrane fractions is not totally surprising since it seems normal that some of the GSP proteins will link the inner-membrane and outer-membrane components of the machinery. The energy-transducing protein TonB, which is involved in the transport of molecules across the outer membrane, has also been found associated with both membranes (Letain \& Postle, 1997). Some properties of ExeB (sequence similarity, topology, a proline-rich segment, a high $\mathrm{pI}$ ) are similar to those of TonB. Although they are rather rich in proline $(9 \%)$, there is no proline domain in OutB or PulB (Fig. 1), their theoretical pIs are $7 \cdot 4$ and $7 \cdot 1$, respectively, and they have no obvious homology with TonB. Thus, the homology between ExeB and TonB may not reflect a general feature of members of the GspB family.

An interesting point of this study is that an outB mutation can be suppressed by the overexpression of outD either from the chromosomal copy or from a plasmid. This observation could explain the discrepancy observed between the phenotypes of exeB, outB and pulB mutants. The effect of the outB mutation on 
pectate lyase secretion was previously tested (Condemine et al., 1992). However, to induce pectate lyase synthesis, galacturonate or polygalacturonate had to be added to the culture medium. Their presence also induced the outC-O operon, increasing the level of OutD synthesis. In these conditions, secretion of pectate lyase was only slightly reduced. When an outB mutant was tested for EGZ secretion, which does not necessitate the addition of an inducer, the outB mutant appeared to be secretion deficient. Aerolysin secretion can be tested in A. bydrophila without addition of an inducer, leaving the expression of the exeC-N operon at its basal level (Jiang \& Howard, 1991). The exeB mutant appears to be deficient for secretion. On the other hand, the $K$. oxytoca pulB mutant did not appear to be affected for pullulanase secretion. The phenotype of the pulB mutant was tested in the presence of maltose, which induced both pullulanase and PulD synthesis. It is probable that if pullulanase synthesis could be made independent from maltose induction, a $p u l B$ mutant would be secretion deficient.

The suppression of the outB phenotype by overexpression of outD led us to look for evidence of interactions between the two proteins. In Erw. chrysanthemi, OutB was stabilized by OutD. Coexpression of OutB and OutD in the same strain increased the quantity of OutD detectable in the bacteria. We have previously used this type of experiment to show the interactions between OutD and OutS, and between OutD and the secreted proteins (Shevchik et al., 1997). However, the OutD stabilization obtained with OutB was less efficient than that observed with OutS or PelB, suggesting that the interaction might be weaker. This lesser effect prevented us from testing the protection by OutB of truncated derivatives of OutD: most of them are unstable and could not be stabilized by OutB to a level allowing for their detection. Protection of OutD by PelB ${ }^{\mathrm{sp}}$-OutB shows that the anchoring of OutB in the inner membrane is not required for the OutB-OutD interaction.

What might the role of OutB be? It has been proposed that ExeB could function with the putative ATPase ExeA to transduce metabolic energy to the opening of the secretion pore (Schoenhofen et al., 1998). However, the presence of OutB and PulB in two strains in which no ExeA homologue has been found and the presence of GspA in Esc. coli that does not contain a GspB make this hypothesis doubtful in the case of OutB. The results presented here suggest that OutB is necessary for the proper functioning of the Out machinery, probably by interaction with OutD. The presence of outB and $p u l B$ next to outS and puls could indicate that the two proteins may collaborate to pilot OutD and PulD to the outer membrane. Elucidation of the role of OutB requires further investigation.

\section{ACKNOWLEDGEMENTS}

We are grateful to Jean-Claude Lazzaroni for plasmid pJBSkpn, to Frédéric Barras for EGZ antibodies, to Nicole Cotte-Pattat, Sylvie Reverchon and Valérie James for reading the manuscript. We thank Janine Robert-Baudouy for her support. This work was supported by grants from the CNRS and the DRED.

\section{REFERENCES}

Andro, T., Chambost, J. P., Kotoujanski, A., Cattaneo, J., Bertheau, Y., Barras, F., van Gijsegem, F. \& Coleno, A. (1984). Mutants of Erwinia chrysanthemi defective in secretion of pectinase and cellulase. J Bacteriol 160, 1199-1203.

Bitter, W., Koster, M., Latijnhouwers, M., de Cock, H. \& Tommassen, J. (1998). Formation of oligomeric rings by XcpQ and PilQ, which are involved in protein transport across the outer membrane of Pseudomonas aeruginosa. Mol Microbiol 27, 209-219.

Bleves, S., Voulhoux, R., Michel, G., Lazdunski, A., Tommassen, J. \& Filloux, A. (1998). The secretion apparatus of Pseudomonas aeruginosa: identification of a fifth pseudopilin, XcpX (GspK family). Mol Microbiol 27, 31-40.

Broome-Smith, J. K., Tadayvon, M. \& Zhang, Y. (1990). $\beta$ Lactamase as a probe of membrane protein assembly and protein export. Mol Microbiol 4, 1637-1644.

Condemine, G. \& Robert-Baudouy, J. (1987). 2-Keto-3-deoxygluconate transport system in Erwinia chrysanthemi. J Bacteriol 169, 1972-1978.

Condemine, G. \& Robert-Baudouy, J. (1995). Synthesis and secretion of Erwinia chrysanthemi virulence factors are coregulated. Mol Plant Microbe Interact 8, 632-636.

Condemine, G., Dorel, C., Hugouvieux-Cotte-Pattat, N. \& RobertBaudouy, J. (1992). Some of the out genes involved in protein secretion of pectate lyases in Erwinia chrysanthemi are regulated by kdgR. Mol Microbiol 6, 3199-3211.

Daefler, S., Guilvout, I., Hardie, K. R., Pugsley, A. P. \& Russel, M. (1997). The C-terminal domain of the secretin PulD contains the binding site for its cognate chaperone, PulS, and confers PulS dependence on pIV ${ }^{\mathrm{p} 1}$ function. Mol Microbiol 24, 465-475.

Dykxhoorn, D. M., St Pierre, R. \& Linn, T. (1996). A set of compatible tac promoter expression vectors. Gene 177, 133-136. d'Enfert, C. \& Pugsley, A. P. (1989). Klebsiella pneumoniae pulS gene encodes an outer membrane lipoprotein required for pullulanase secretion. J Bacteriol 171, 3673-3679.

Francetic, O. \& Pugsley, A. P. (1996). The cryptic general secretory pathway ( $g s p)$ operon of Escherichia coli K-12 encodes functional proteins. J Bacteriol 178, 3544-3549.

Hardie, K. R., Lory, S. \& Pugsley, A. P. (1996). Insertion of an outer membrane protein in Escherichia coli requires a chaperone-like protein. EMBO J 15, 978-988.

Howard, S. P., Meiklejohn, H. G., Shivak, D. \& Jahagirdar, R. (1996). A TonB-like protein and a novel membrane protein containing an ATP-binding cassette function together in exotoxin secretion. Mol Microbiol 22, 595-604.

Hugouvieux-Cotte-Pattat, N. \& Robert-Baudouy, J. (1985). Lactose metabolism in Erwinia chrysanthemi. J Bacteriol 129, 1223-1231.

Jahagirdar, R. \& Howard, S. P. (1994). Isolation and characterization of a second exe operon required for extracellular protein secretion in Aeromonas hydrophila. J Bacteriol 176, 6819-6826.

Jiang, B. \& Howard, S. P. (1991). Mutagenesis and isolation of Aeromonas hydrophila genes which are required for extracellular secretion. J Bacteriol 173, 1241-1249.

Johnson, B. H. \& Hecht, M. H. (1994). Recombinant proteins can 
be isolated from E. coli by repeated cycles of freezing and thawing. Bio/Technology 12, 1357-1360.

Kagami, Y., Ratliff, M., Surber, M., Martinez, A. \& Nunn, D. N. (1998). Type II protein secretion by Pseudomonas aeruginosa: genetic suppression of a condition of a conditional mutation in the pilin-like component XcpT by the cytoplasmic component XcpR. Mol Microbiol 27, 221-233.

Kyte, J. \& Doolittle, R. F. (1982). A simple method for displaying the hydrophobic character of a protein. J Mol Biol 157, 105-132.

Laemmli, U. K. (1970). Cleavage of structural proteins during assembly of the head of bacteriophage T4. Nature 227, 680-685.

Letain, T. E. \& Postle, K. (1997). TonB protein appears to transduce energy by shuttling between the cytoplasmic membrane and the outer membrane in Escherichia coli. Mol Microbiol 24, 271-283.

Lindeberg, M. \& Collmer, A. (1992). Analysis of eight genes in a cluster required for pectic enzyme secretion by Erwinia chrysanthemi: sequence comparison with secretion genes from other Gram-negative bacteria. J Bacteriol 174, 7385-7397.

Lindeberg, M., Salmond, G. P. C. \& Collmer, A. (1996). Complementation of deletion mutations in a cloned functional cluster of Erwinia chrysanthemi out genes with Erwinia carotovora out homologues reveals OutC and OutD as candidate gatekeepers of species-specific secretion via the type II pathway. Mol Microbiol 20, 175-190.

Linderoth, N. A., Simon, M. N. \& Russel, M. (1997). The filamentous phage pIV multimer visualized by scanning transmission electron microscopy. Science 278, 1635-1638.

Michel, G., Bleves, S., Ball, G., Lazdunski, A. \& Filloux, A. (1998). Mutual stabilization between XcpZ and XcpY components of the secretory apparatus in Pseudomonas aeruginosa. Microbiology 144, 3379-3386.

Miller, J. H. (1972). Experiments in Molecular Genetics. Cold Spring Harbor, NY: Cold Spring Harbor Laboratory.

Nunn, D. N. \& Lory, S. (1991). Product of the Pseudomonas aeruginosa gene pilD is a prepilin leader peptidase. Proc Natl Acad Sci USA 88, 3281-3285.

Osborn, M. J., Gander, J. E., Parisi, E. \& Carson, J. (1972). Mechanism of assembly of the outer membrane of Salmonella typhimurium. J Biol Chem 247, 3962-3972.

Possot, O. M., Gérard-Vincent, M. \& Pugsley, A. P. (1999). Membrane association and multimerization of secretion component PulC. J Bacteriol 181, 4004-4011.

Pugsley, A. P. (1993). The complete general secretory pathway in Gram-negative bacteria. Microbiol Rev 57, 50-108.

Pugsley, A. P. \& Possot, O. (1993). The general secretory pathway of Klebsiella oxytoca: no evidence for relocalization or assembly of pilin-like PulG protein into a multiprotein complex. Mol Microbiol 10, 665-674.

Py, B., Loiseau, L. \& Barras, F. (1999). Assembly of the type II secretion machinery of Erwinia chrysanthemi: direct interaction and associated conformational change between OutE, the putative ATP-binding component and the membrane protein OutL. $J$ Mol Biol 289, 659-670.
Reeves, P. J., Douglas, P. \& Salmond, G. P. C. (1994). The general secretion pathway of Erwinia carotovora subsp. carotovora: analysis of the cytoplasmic membrane topology of seven Out proteins using a $\beta$-lactamase topology probe. Mol Microbiol 12, 445-457.

Reverchon, S., Nasser, W. \& Robert-Baudouy, J. (1994). pecS : a locus controlling pectinase, cellulase and blue pigment production in Erwinia chrysanthemi. Mol Microbiol 11, 1127-1139.

Russel, M. \& Model, P. (1984). Replacement of the fip gene of Escherichia coli by an inactive gene cloned on a plasmid. J Bacteriol 159, 1034-1039.

Sambrook, J., Fritsch, E. F. \& Maniatis, T. (1989). Molecular Cloning: a Laboratory Manual, 2nd edn. Cold Spring Harbor, NY : Cold Spring Harbor Laboratory.

Sandkvist, M., Bagdasarian, M., Howard, S.P. \& DiRita, V. J. (1995). Interaction between the autokinase EspE and EspL in the cytoplasmic membrane is required for extracellular secretion in Vibrio cholerae. EMBO J 14, 1664-1673.

Sandkvist, M., Hough, L. P., Bagdasarian, M. M. \& Bagdasarian, M. (1999). Direct interaction of the EpsL and EpsM proteins of the general secretion apparatus in Vibrio cholerae. J Bacteriol 181, 3129-3135.

Schmidt, H., Henkel, B. \& Karch, H. (1997). A gene cluster closely related to type II secretion pathway operons of Gram-negative bacteria is located on the large plasmid of enterohemorrhagic coli Escherichia coli $\mathrm{O} 157$ strains. FEMS Microbiol Lett 148, 265-272.

Schoenhofen, I. C., Stratilo, C. \& Howard, S. P. (1998). An ExeAB complex in the type II secretion pathway of Aeromonas hydrophila: effect of ATP-binding cassette mutations on complex formation and function. Mol Microbiol 29, 1237-1247.

Shevchik, V. E. \& Condemine, G. (1998). Functional characterization of the Erwinia chrysanthemi OutS protein, an element of a type II secretion system. Microbiology 144, 3219-3228.

Shevchik, V. E., Condemine, G., Hugouvieux-Cotte-Pattat, N. \& Robert-Baudouy, J. (1996). Characterizaton of pectin methylesterase B, an outer membrane lipoprotein of Erwinia chrysanthemi. Mol Microbiol 19, 455-466.

Shevchik, V. E., Robert-Baudouy, J. \& Condemine, G. (1997). Specific interaction between OutD, an Erwinia chrysanthemi outer membrane protein of the general secretory pathway and secreted proteins. EMBO J 16, 3007-3016.

Tabor, S. \& Richardson, C. (1985). A bacteriophage T7 RNA polymerase/promoter system for controlled exclusive expression of specific genes. Proc Natl Acad Sci USA 82, 1074-1078.

Thomas, J. D., Reeves, P. J. \& Salmond, G. P. C. (1997). The general secretion pathway of Erwinia carotovora subsp. carotovora: analysis of the membrane topology of OutC and OutF. Microbiology 143, 713-720.

Wood, P. J. (1980). Specificity in the interaction of direct dyes with polysaccharides. Carbohydrate Res 85, 271-287.

Received 18 October 1999; accepted 22 November 1999. 